\title{
ON PELL HYBRINOMIALS
}

\author{
MIROSŁAW LIANA, ANETTA SZYNAL-LIANA, AND IWONA WŁOCH
}

Received 21 May, 2019

\begin{abstract}
Hybrid numbers generalize complex, hyperbolic and dual numbers, simultaneously. Special kinds of hybrid numbers, related to numbers of Fibonacci type, among others Pell numbers, were introduced quite recently. In this paper we introduce and study polynomials, which are a generalization of Pell hybrid numbers and so called Pell hybrinomials.
\end{abstract}

2010 Mathematics Subject Classification: 11B37; 11B39; 97F50

Keywords: Pell numbers, complex numbers, hyperbolic numbers, dual numbers, polynomials

\section{INTRODUCTION}

Pell numbers are well-known numbers in the number theory and they belong to the wide class of numbers of the Fibonacci type. The $n$th Pell number $P_{n}$ is defined recursively by the second order linear recurrence relation $P_{n}=2 P_{n-1}+P_{n-2}$, for $n \geq 2$ with initial conditions $P_{0}=0, P_{1}=1$. A special version of Pell numbers is Pell-Lucas numbers $Q_{n}$ (also named as companion Pell numbers). Then $Q_{n}=$ $2 Q_{n-1}+Q_{n-2}$, for $n \geq 2$ with $Q_{0}=Q_{1}=2$.

Distinct properties of Pell and Pell-Lucas numbers can be found for example in $[1,2,5]$. In [3] Horadam and Mahon introduced Pell and Pell-Lucas polynomials as follows.

For any variable quantity $x$, the Pell polynomial $P_{n}(x)$ is defined as $P_{n}(x)=$ $2 x \cdot P_{n-1}(x)+P_{n-2}(x)$ for $n \geq 2$ with $P_{0}(x)=0, P_{1}(x)=1$.

The Pell-Lucas polynomial $Q_{n}(x)$ is defined as $Q_{n}(x)=2 x \cdot Q_{n-1}(x)+Q_{n-2}(x)$ for $n \geq 2$ with initial terms $Q_{0}(x)=2, Q_{1}(x)=2 x$.

For $x=1$ we obtain Pell and Pell-Lucas numbers, respectively.

For any $x$ let $\alpha(x)=x+\sqrt{x^{2}+1}$ and $\beta(x)=x-\sqrt{x^{2}+1}$. Then solving secondorder linear recurrence relations, for $P_{n}(x)$ and $Q_{n}(x)$, respectively, we have

$$
P_{n}(x)=\frac{\alpha^{n}(x)-\beta^{n}(x)}{\alpha(x)-\beta(x)}
$$

and

$$
Q_{n}(x)=\alpha^{n}(x)+\beta^{n}(x) .
$$


One of the generalizations of the Pell polynomial is the Horadam polynomial, whose properties can be found in [4].

Hybrid numbers were introduced by Özdemir in [6] as a new generalization of complex, hyperbolic and dual numbers.

Let $\mathbb{K}$ be the set of hybrid numbers $\mathbf{Z}$ of the form

$$
\mathbf{Z}=a+b \mathbf{i}+c \varepsilon+d \mathbf{h},
$$

where $a, b, c, d \in \mathbb{R}$ and $\mathbf{i}, \varepsilon, \mathbf{h}$ are operators such that

$$
\mathbf{i}^{2}=-1, \varepsilon^{2}=0, \mathbf{h}^{2}=1
$$

and

$$
\mathbf{i h}=-\mathbf{h i}=\varepsilon+\mathbf{i} .
$$

If $\mathbf{Z}_{1}=a_{1}+b_{1} \mathbf{i}+c_{1} \varepsilon+d_{1} \mathbf{h}$, and $\mathbf{Z}_{2}=a_{2}+b_{2} \mathbf{i}+c_{2} \varepsilon+d_{2} \mathbf{h}$, are any two hybrid numbers then equality, addition, substraction and multiplication by scalar are defined.

Equality: $\mathbf{Z}_{1}=\mathbf{Z}_{2}$ only if $a_{1}=a_{2}, b_{1}=b_{2}, c_{1}=c_{2}, d_{1}=d_{2}$,

addition: $\mathbf{Z}_{1}+\mathbf{Z}_{2}=\left(a_{1}+a_{2}\right)+\left(b_{1}+b_{2}\right) \mathbf{i}+\left(c_{1}+c_{2}\right) \varepsilon+\left(d_{1}+d_{2}\right) \mathbf{h}$, substraction: $\mathbf{Z}_{1}-\mathbf{Z}_{2}=\left(a_{1}-a_{2}\right)+\left(b_{1}-b_{2}\right) \mathbf{i}+\left(c_{1}-c_{2}\right) \varepsilon+\left(d_{1}-d_{2}\right) \mathbf{h}$, multiplication by scalar $s \in \mathbb{R}: s \mathbf{Z}_{1}=s a_{1}+s b_{1} \mathbf{i}+s c_{1} \varepsilon+s d_{1} \mathbf{h}$.

The hybrid numbers multiplication is defined using (1.3) and (1.4). Note that using formulas (1.3) and (1.4) we can find the product of any two hybrid units. The following Table presents products of $\mathbf{i}, \varepsilon$, and $\mathbf{h}$ Using rules given in Table 1. the

TABLE 1. The hybrid number multiplication.

\begin{tabular}{|c||c|c|c|}
\hline$\cdot$ & $\mathbf{i}$ & $\varepsilon$ & $\mathbf{h}$ \\
\hline \hline $\mathbf{i}$ & -1 & $1-\mathbf{h}$ & $\varepsilon+\mathbf{i}$ \\
\hline$\varepsilon$ & $\mathbf{h}+1$ & 0 & $-\varepsilon$ \\
\hline $\mathbf{h}$ & $-\varepsilon-\mathbf{i}$ & $\varepsilon$ & 1 \\
\hline
\end{tabular}

multiplication of hybrid numbers can be made analogously as multiplications of algebraic expressions. For hybrid numbers details, see [6].

A special kind of hybrid numbers, namely Pell hybrid numbers and Pell-Lucas hybrid numbers, were introduced in [7] as follows.

The $n$th Pell hybrid number $P H_{n}$ and the $n$th Pell-Lucas hybrid number $Q H_{n}$ are defined as

$$
\begin{gathered}
P H_{n}=P_{n}+\mathbf{i} P_{n+1}+\varepsilon P_{n+2}+\mathbf{h} P_{n+3}, \\
Q H_{n}=Q_{n}+\mathbf{i} Q_{n+1}+\varepsilon Q_{n+2}+\mathbf{h} Q_{n+3},
\end{gathered}
$$

respectively.

Interesting results of Pell and Pell-Lucas hybrid numbers obtained recently can be found in [8]. 
In this paper we introduce Pell and Pell-Lucas hybrinomials, i.e. polynomials, which are a generalization of Pell hybrid numbers and Pell-Lucas hybrid numbers, respectively.

For $n \geq 0$ Pell and Pell-Lucas hybrinomials are defined by

$$
P H_{n}(x)=P_{n}(x)+\mathbf{i} P_{n+1}(x)+\varepsilon P_{n+2}(x)+\mathbf{h} P_{n+3}(x)
$$

and

$$
Q H_{n}(x)=Q_{n}(x)+\mathbf{i} Q_{n+1}(x)+\varepsilon Q_{n+2}(x)+\mathbf{h} Q_{n+3}(x),
$$

where $P_{n}(x)$ is the $n$th Pell polynomial, $Q_{n}(x)$ is the the $n$-th Pell-Lucas polynomial and $\mathbf{i}, \varepsilon, \mathbf{h}$ are hybrid units satisfy (1.3) and (1.4).

For $x=1$ we obtain Pell hybrid numbers and Pell-Lucas hybrid numbers, respectively.

\section{Properties of Pell and Pell-Lucas hybrinomials}

Theorem 1. Let $n \geq 0$ be an integer. For any variable quantity $x$, we have

$$
P H_{n}(x)=2 x \cdot P H_{n-1}(x)+P H_{n-2}(x) \text { for } n \geq 2
$$

with $P H_{0}(x)=\mathbf{i}+\varepsilon \cdot(2 x)+\mathbf{h} \cdot\left(4 x^{2}+1\right)$

and $P H_{1}(x)=1+\mathbf{i} \cdot(2 x)+\varepsilon \cdot\left(4 x^{2}+1\right)+\mathbf{h} \cdot\left(8 x^{3}+4 x\right)$.

Proof. If $n=2$ we have

$$
\begin{aligned}
\mathrm{PH}_{2}(x)= & 2 x \cdot P H_{1}(x)+P H_{0}(x) \\
= & 2 x \cdot\left(1+\mathbf{i} \cdot(2 x)+\varepsilon \cdot\left(4 x^{2}+1\right)+\mathbf{h} \cdot\left(8 x^{3}+4 x\right)\right) \\
& +\mathbf{i}+\varepsilon \cdot(2 x)+\mathbf{h} \cdot\left(4 x^{2}+1\right) \\
= & 2 x+\mathbf{i} \cdot\left(4 x^{2}+1\right)+\varepsilon \cdot\left(8 x^{3}+4 x\right)+\mathbf{h} \cdot\left(16 x^{4}+12 x^{2}+1\right) \\
= & P_{2}(x)+\mathbf{i} P_{3}(x)+\varepsilon P_{4}(x)+\mathbf{h} P_{5}(x) .
\end{aligned}
$$

If $n \geq 3$ then using the definition of Pell polynomials we have

$$
\begin{aligned}
P H_{n}(x)= & P_{n}(x)+\mathbf{i} P_{n+1}(x)+\varepsilon P_{n+2}(x)+\mathbf{h} P_{n+3}(x) \\
= & \left(2 x \cdot P_{n-1}(x)+P_{n-2}(x)\right)+\mathbf{i}\left(2 x \cdot P_{n}(x)+P_{n-1}(x)\right) \\
& +\varepsilon\left(2 x \cdot P_{n+1}(x)+P_{n}(x)\right)+\mathbf{h}\left(2 x \cdot P_{n+2}(x)+P_{n+1}(x)\right) \\
= & 2 x\left(P_{n-1}(x)+\mathbf{i} \cdot P_{n}(x)+\varepsilon \cdot P_{n+1}(x)+\mathbf{h} \cdot P_{n+2}(x)\right) \\
& +P_{n-2}(x)+\mathbf{i} \cdot P_{n-1}(x)+\varepsilon \cdot P_{n}(x)+\mathbf{h} \cdot P_{n+1}(x) \\
= & 2 x \cdot P H_{n-1}(x)+P H_{n-2}(x),
\end{aligned}
$$

which ends the proof.

In the same way one can easily prove the next theorem. 
Theorem 2. Let $n \geq 0$ be an integer. For any variable quantity $x$, we have

$$
Q H_{n}(x)=2 x \cdot Q H_{n-1}(x)+Q H_{n-2}(x) \text { for } n \geq 2
$$

with $Q H_{0}(x)=2+\mathbf{i} \cdot(2 x)+\varepsilon \cdot\left(4 x^{2}+2\right)+\mathbf{h} \cdot\left(8 x^{3}+6 x\right)$ and $Q H_{1}(x)=2 x+\mathbf{i}$. $\left(4 x^{2}+2\right)+\varepsilon \cdot\left(8 x^{3}+6 x\right)+\mathbf{h} \cdot\left(16 x^{4}+16 x^{2}+2\right)$.

Now we give so called Binet formulas for Pell and Pell-Lucas hybrinomials.

Theorem 3. Let $n \geq 0$ be an integer. Then

$$
\begin{aligned}
P H_{n}(x)= & \frac{\alpha^{n}(x)}{\alpha(x)-\beta(x)}\left(1+\mathbf{i} \alpha(x)+\varepsilon \alpha^{2}(x)+\mathbf{h} \alpha^{3}(x)\right) \\
& -\frac{\beta^{n}(x)}{\alpha(x)-\beta(x)}\left(1+\mathbf{i} \beta(x)+\varepsilon \beta^{2}(x)+\mathbf{h} \beta^{3}(x)\right),
\end{aligned}
$$

where $\alpha(x)=x+\sqrt{x^{2}+1}$ and $\beta(x)=x-\sqrt{x^{2}+1}$.

Proof. Using (1.1), (1.5) and (1.7) we have

$$
\begin{aligned}
P H_{n}(x)= & P_{n}(x)+\mathbf{i} P_{n+1}(x)+\varepsilon P_{n+2}(x)+\mathbf{h} P_{n+3}(x) \\
= & \frac{\alpha^{n}(x)-\beta^{n}(x)}{\alpha(x)-\beta(x)}+\mathbf{i} \frac{\alpha^{n+1}(x)-\beta^{n+1}(x)}{\alpha(x)-\beta(x)} \\
& +\varepsilon \frac{\alpha^{n+2}(x)-\beta^{n+2}(x)}{\alpha(x)-\beta(x)}+\mathbf{h} \frac{\alpha^{n+3}(x)-\beta^{n+3}(x)}{\alpha(x)-\beta(x)}
\end{aligned}
$$

and after calculations the result follows.

In the same way, using (1.2), (1.6) and (1.8), one can easily prove the next theorem.

Theorem 4. Let $n \geq 0$ be an integer. Then

$$
\begin{aligned}
Q H_{n}(x)= & \alpha^{n}(x)\left(1+\mathbf{i} \alpha(x)+\varepsilon \alpha^{2}(x)+\mathbf{h} \alpha^{3}(x)\right) \\
& +\beta^{n}(x)\left(1+\mathbf{i} \beta(x)+\varepsilon \beta^{2}(x)+\mathbf{h} \beta^{3}(x)\right),
\end{aligned}
$$

where $\alpha(x)=x+\sqrt{x^{2}+1}$ and $\beta(x)=x-\sqrt{x^{2}+1}$.

Now we will give some identities related to the well-known identities for classical Pell numbers

(Catalan identity) $P_{n-r} \cdot P_{n+r}-\left(P_{n}\right)^{2}=(-1)^{n-r+1} P_{r}^{2}$,

(Cassini identity) $P_{n-1} \cdot P_{n+1}-\left(P_{n}\right)^{2}=(-1)^{n}$,

(d'Ocagne identity) $P_{m} \cdot P_{n+1}-P_{m+1} \cdot P_{n}=(-1)^{n} P_{m-n}$.

We give their versions for Pell and Pell-Lucas hybrinomials. These identities can be proved using Binet formulas.

For simplicity of notation let $\Delta(x)=\alpha(x)-\beta(x)$, 
$\hat{\alpha}(x)=1+\mathbf{i} \alpha(x)+\varepsilon \alpha^{2}(x)+\mathbf{h} \alpha^{3}(x)$, $\hat{\beta}(x)=1+\mathbf{i} \beta(x)+\varepsilon \beta^{2}(x)+\mathbf{h} \beta^{3}(x)$.

Then we can write (2.3) and (2.4) as $P H_{n}(x)=\frac{\alpha^{n}(x)}{\Delta(x)} \hat{\alpha}(x)-\frac{\beta^{n}(x)}{\Delta(x)} \hat{\beta}(x)$

and

$Q H_{n}(x)=\alpha^{n}(x) \hat{\alpha}(x)+\beta^{n}(x) \hat{\beta}(x)$, respectively.

Moreover, $\alpha(x) \cdot \beta(x)=-1$ and $\Delta^{2}(x)=4 x^{2}+4$.

Theorem 5 (Catalan identity for Pell hybrinomials). Let $n \geq 0, r \geq 0$ be integers such that $n \geq r$. Then

$$
\begin{aligned}
& P H_{n-r}(x) \cdot P H_{n+r}(x)-\left(P H_{n}(x)\right)^{2} \\
& =\frac{(-1)^{n}}{4 x^{2}+4} \hat{\alpha}(x) \hat{\beta}(x)\left(1-\frac{\beta^{r}(x)}{\alpha^{r}(x)}\right)+\frac{(-1)^{n}}{4 x^{2}+4} \hat{\beta}(x) \hat{\alpha}(x)\left(1-\frac{\alpha^{r}(x)}{\beta^{r}(x)}\right) .
\end{aligned}
$$

Proof. For integers $n \geq 0, r \geq 0$ and $n \geq r$ we have

$$
\begin{aligned}
P & H_{n-r}(x) \cdot P H_{n+r}(x)-\left(P H_{n}(x)\right)^{2} \\
= & \left(\frac{\alpha^{n-r}(x)}{\Delta(x)} \hat{\alpha}(x)-\frac{\beta^{n-r}(x)}{\Delta(x)} \hat{\beta}(x)\right) \cdot\left(\frac{\alpha^{n+r}(x)}{\Delta(x)} \hat{\alpha}(x)-\frac{\beta^{n+r}(x)}{\Delta(x)} \hat{\beta}(x)\right) \\
& -\left(\frac{\alpha^{n}(x)}{\Delta(x)} \hat{\alpha}(x)-\frac{\beta^{n}(x)}{\Delta(x)} \hat{\beta}(x)\right) \cdot\left(\frac{\alpha^{n}(x)}{\Delta(x)} \hat{\alpha}(x)-\frac{\beta^{n}(x)}{\Delta(x)} \hat{\beta}(x)\right) \\
= & -\frac{\alpha^{n-r}(x)}{\Delta(x)} \hat{\alpha}(x) \frac{\beta^{n+r}(x)}{\Delta(x)} \hat{\beta}(x)-\frac{\beta^{n-r}(x)}{\Delta(x)} \hat{\beta}(x) \frac{\alpha^{n+r}(x)}{\Delta(x)} \hat{\alpha}(x) \\
& +\frac{\alpha^{n}(x)}{\Delta(x)} \hat{\alpha}(x) \frac{\beta^{n}(x)}{\Delta(x)} \hat{\beta}(x)+\frac{\beta^{n}(x)}{\Delta(x)} \hat{\beta}(x) \frac{\alpha^{n}(x)}{\Delta(x)} \hat{\alpha}(x) \\
= & -\frac{\alpha^{n-r}(x) \beta^{n+r}(x)}{\Delta^{2}(x)} \hat{\alpha}(x) \hat{\beta}(x)-\frac{\beta^{n-r}(x) \alpha^{n+r}(x)}{\Delta^{2}(x)} \hat{\beta}(x) \hat{\alpha}(x) \\
& +\frac{\alpha^{n}(x) \beta^{n}(x)}{\Delta^{2}(x)} \hat{\alpha}(x) \hat{\beta}(x)+\frac{\beta^{n}(x) \alpha^{n}(x)}{\Delta^{2}(x)} \hat{\beta}(x) \hat{\alpha}(x) \\
= & \frac{\alpha^{n}(x) \beta^{n}(x)}{\Delta^{2}(x)} \hat{\alpha}(x) \hat{\beta}(x)\left(1-\frac{\beta^{r}(x)}{\alpha^{r}(x)}\right)+\frac{\alpha^{n}(x) \beta^{n}(x)}{\Delta^{2}(x)} \hat{\beta}(x) \hat{\alpha}(x)\left(1-\frac{\alpha^{r}(x)}{\beta^{r}(x)}\right) \\
= & \frac{(-1)^{n}}{4 x^{2}+4} \hat{\alpha}(x) \hat{\beta}(x)\left(1-\frac{\beta^{r}(x)}{\alpha^{r}(x)}\right)+\frac{(-1)^{n}}{4 x^{2}+4} \hat{\beta}(x) \hat{\alpha}(x)\left(1-\frac{\alpha^{r}(x)}{\beta^{r}(x)}\right),
\end{aligned}
$$

which ends the proof.

In the same way one can easily prove the next theorem, which gives Catalan identity for Pell-Lucas hybrinomials. 
Theorem 6 (Catalan identity for Pell-Lucas hybrinomials). Let $n \geq 0, r \geq 0$ be integers such that $n \geq r$. Then

$$
\begin{aligned}
& Q H_{n-r}(x) \cdot Q H_{n+r}(x)-\left(Q H_{n}(x)\right)^{2} \\
& =(-1)^{n} \hat{\alpha}(x) \hat{\beta}(x)\left(\frac{\beta^{r}(x)}{\alpha^{r}(x)}-1\right)+(-1)^{n} \hat{\beta}(x) \hat{\alpha}(x)\left(\frac{\alpha^{r}(x)}{\beta^{r}(x)}-1\right) .
\end{aligned}
$$

Note that for $r=1$ we get Cassini identities for Pell and Pell-Lucas hybrinomials. Moreover, for $r=1$ we have

$1-\frac{\beta(x)}{\alpha(x)}=\frac{\alpha(x)-\beta(x)}{\alpha(x)}=\frac{\Delta(x)}{\alpha(x)}$ and $1-\frac{\alpha(x)}{\beta(x)}=\frac{\beta(x)-\alpha(x)}{\beta(x)}=-\frac{\Delta(x)}{\beta(x)}$.

Corollary 1 (Cassini identities for Pell and Pell-Lucas hybrinomials). Let $n \geq 0$ be an integer. Then

$$
\begin{gathered}
P H_{n-1}(x) \cdot P H_{n+1}(x)-\left(P H_{n}(x)\right)^{2} \\
=\frac{(-1)^{n-1} \beta(x)}{\Delta(x)} \hat{\alpha}(x) \hat{\beta}(x)-\frac{(-1)^{n-1} \alpha(x)}{\Delta(x)} \hat{\beta}(x) \hat{\alpha}(x) . \\
Q H_{n-1}(x) \cdot Q H_{n+1}(x)-\left(Q H_{n}(x)\right)^{2} \\
=(-1)^{n} \hat{\alpha}(x) \hat{\beta}(x)\left(\frac{\beta(x)}{\alpha(x)}-1\right)+(-1)^{n} \hat{\beta}(x) \hat{\alpha}(x)\left(\frac{\alpha(x)}{\beta(x)}-1\right) .
\end{gathered}
$$

Theorem 7 (d'Ocagne identity for Pell hybrinomials). Let $m \geq 0, n \geq 0$ be integers such that $m \geq n$. Then

$$
\begin{aligned}
& P H_{m}(x) \cdot P H_{n+1}(x)-P H_{m+1}(x) \cdot P H_{n}(x) \\
& =\frac{(-1)^{n} \alpha^{m-n}(x)}{\Delta(x)} \hat{\alpha}(x) \hat{\beta}(x)-\frac{(-1)^{n} \beta^{m-n}(x)}{\Delta(x)} \hat{\beta}(x) \hat{\alpha}(x) .
\end{aligned}
$$

Proof. Let $m, n$ be as in the statement of the Theorem. Then

$$
\begin{aligned}
P & H_{m}(x) \cdot P H_{n+1}(x)-P H_{m+1}(x) \cdot P H_{n}(x) \\
= & \left(\frac{\alpha^{m}(x)}{\Delta(x)} \hat{\alpha}(x)-\frac{\beta^{m}(x)}{\Delta(x)} \hat{\beta}(x)\right) \cdot\left(\frac{\alpha^{n+1}(x)}{\Delta(x)} \hat{\alpha}(x)-\frac{\beta^{n+1}(x)}{\Delta(x)} \hat{\beta}(x)\right) \\
& -\left(\frac{\alpha^{m+1}(x)}{\Delta(x)} \hat{\alpha}(x)-\frac{\beta^{m+1}(x)}{\Delta(x)} \hat{\beta}(x)\right) \cdot\left(\frac{\alpha^{n}(x)}{\Delta(x)} \hat{\alpha}(x)-\frac{\beta^{n}(x)}{\Delta(x)} \hat{\beta}(x)\right) \\
= & \frac{\alpha^{m+n+1}(x)}{\Delta^{2}(x)} \hat{\alpha}^{2}(x)-\frac{\alpha^{m}(x) \beta^{n+1}(x)}{\Delta^{2}(x)} \hat{\alpha}(x) \hat{\beta}(x)-\frac{\alpha^{n+1}(x) \beta^{m}(x)}{\Delta^{2}(x)} \hat{\beta}(x) \hat{\alpha}(x) \\
& +\frac{\beta^{m+n+1}(x)}{\Delta^{2}(x)} \hat{\beta}^{2}(x)-\frac{\alpha^{m+1+n}(x)}{\Delta^{2}(x)} \hat{\alpha}^{2}(x)+\frac{\alpha^{m+1}(x) \beta^{n}(x)}{\Delta^{2}(x)} \hat{\alpha}(x) \hat{\beta}(x) \\
& +\frac{\alpha^{n}(x) \beta^{m+1}(x)}{\Delta^{2}(x)} \hat{\beta}(x) \hat{\alpha}(x)-\frac{\beta^{m+1+n}(x)}{\Delta^{2}(x)} \hat{\beta}^{2}(x)
\end{aligned}
$$




$$
\begin{aligned}
= & \frac{\alpha^{m+1}(x) \beta^{n}(x)-\alpha^{m}(x) \beta^{n+1}(x)}{\Delta^{2}(x)} \hat{\alpha}(x) \hat{\beta}(x) \\
& +\frac{\alpha^{n}(x) \beta^{m+1}(x)-\alpha^{n+1}(x) \beta^{m}(x)}{\Delta^{2}(x)} \hat{\beta}(x) \hat{\alpha}(x) \\
= & \frac{\alpha^{m}(x) \beta^{n}(x)(\alpha(x)-\beta(x))}{\Delta^{2}(x)} \hat{\alpha}(x) \hat{\beta}(x) \\
& +\frac{\alpha^{n}(x) \beta^{m}(x)(\beta(x)-\alpha(x))}{\Delta^{2}(x)} \hat{\beta}(x) \hat{\alpha}(x) \\
= & \frac{\alpha^{m}(x) \beta^{n}(x)}{\Delta(x)} \hat{\alpha}(x) \hat{\beta}(x)-\frac{\alpha^{n}(x) \beta^{m}(x)}{\Delta(x)} \hat{\beta}(x) \hat{\alpha}(x) \\
= & \frac{(-1)^{n} \alpha^{m-n}(x)}{\Delta(x)} \hat{\alpha}(x) \hat{\beta}(x)-\frac{(-1)^{n} \beta^{m-n}(x)}{\Delta(x)} \hat{\beta}(x) \hat{\alpha}(x) .
\end{aligned}
$$

Thus the Theorem is proved.

In the same way we can prove next theorems.

Theorem 8 (d'Ocagne identity for Pell-Lucas hybrinomials). Let $m \geq 0, n \geq 0$ be integers such that $m \geq n$. Then

$$
\begin{aligned}
& Q H_{m}(x) \cdot Q H_{n+1}(x)-Q H_{m+1}(x) \cdot Q H_{n}(x) \\
& =(-1)^{n} \beta^{m-n}(x) \Delta(x) \hat{\beta}(x) \hat{\alpha}(x)-(-1)^{n} \alpha^{m-n}(x) \Delta(x) \hat{\alpha}(x) \hat{\beta}(x) .
\end{aligned}
$$

Theorem 9. Let $m \geq 0, n \geq 0$ be integers. Then

$$
\begin{aligned}
& P H_{m}(x) \cdot Q H_{n}(x)-Q H_{m}(x) \cdot P H_{n}(x) \\
& =\frac{2(-1)^{n} \alpha^{m-n}(x)}{\Delta(x)} \hat{\alpha}(x) \hat{\beta}(x)-\frac{2(-1)^{n} \beta^{m-n}(x)}{\Delta(x)} \hat{\beta}(x) \hat{\alpha}(x) .
\end{aligned}
$$

Some identities for Pell and Pell-Lucas hybrinomials can be found by analogy to well-known identities for the Pell and Pell-Lucas polynomials. In the next part of this paper we indicate such identities.

Theorem 10 ([3]). Let $n \geq 1$ be an integer. Then

$$
P_{n+1}(x)+P_{n-1}(x)=Q_{n}(x)=2 x \cdot P_{n}(x)+2 P_{n-1}(x) .
$$

Theorem 11. Let $n \geq 1$ be an integer. Then

$$
P H_{n+1}(x)+P H_{n-1}(x)=Q H_{n}(x)=2 x \cdot P H_{n}(x)+2 P H_{n-1}(x) .
$$


Proof. Using (2.5) we have

$$
\begin{aligned}
P & H_{n+1}(x)+P H_{n-1}(x) \\
= & P_{n+1}(x)+\mathbf{i} P_{n+2}(x)+\varepsilon P_{n+3}(x)+\mathbf{h} P_{n+4}(x) \\
& +P_{n-1}(x)+\mathbf{i} P_{n}(x)+\varepsilon P_{n+1}(x)+\mathbf{h} P_{n+2}(x) \\
= & \left(P_{n+1}(x)+P_{n-1}(x)\right)+\mathbf{i}\left(P_{n+2}(x)+P_{n}(x)\right) \\
& +\varepsilon\left(P_{n+3}(x)+P_{n+1}(x)\right)+\mathbf{h}\left(P_{n+4}(x)+P_{n+2}(x)\right) \\
= & Q_{n}(x)+\mathbf{i} Q_{n+1}(x)+\varepsilon Q_{n+2}(x)+\mathbf{h} Q_{n+3}(x) \\
= & Q H_{n}(x) .
\end{aligned}
$$

On the other hand

$$
\begin{array}{rl}
2 x & P H_{n}(x)+2 P H_{n-1}(x) \\
= & 2 x \cdot\left(P_{n}(x)+\mathbf{i} P_{n+1}(x)+\varepsilon P_{n+2}(x)+\mathbf{h} P_{n+3}(x)\right) \\
& +2\left(P_{n-1}(x)+\mathbf{i} P_{n}(x)+\varepsilon P_{n+1}(x)+\mathbf{h} P_{n+2}(x)\right) \\
= & \left(2 x \cdot P_{n}(x)+P_{n-1}(x)\right)+\mathbf{i}\left(2 x \cdot P_{n+1}(x)+P_{n}(x)\right) \\
& +\varepsilon\left(2 x \cdot P_{n+2}(x)+P_{n+1}(x)\right)+\mathbf{h}\left(2 x \cdot P_{n+3}(x)+P_{n+2}(x)\right) \\
= & Q_{n}(x)+\mathbf{i} Q_{n+1}(x)+\varepsilon Q_{n+2}(x)+\mathbf{h} Q_{n+3}(x) \\
= & Q H_{n}(x),
\end{array}
$$

so the result follows.

Theorem 12 ([3]). Let $n \geq 1$ be an integer. Then

$$
Q_{n+1}(x)+Q_{n-1}(x)=4\left(x^{2}+1\right) P_{n}(x) .
$$

Theorem 13. Let $n \geq 1$ be an integer. Then

$$
Q H_{n+1}(x)+Q H_{n-1}(x)=4\left(x^{2}+1\right) P H_{n}(x) .
$$

Proof. Using (2.6) and proceeding in the same way as in the Theorem 11 the result follows.

Theorem 14 ([3]). Let $n \geq 2$ be an integer. Then

$$
\sum_{l=1}^{n-1} P_{l}(x)=\frac{P_{n}(x)+P_{n-1}(x)-1}{2 x} .
$$

Theorem 15. Let $n \geq 2$ be an integer. Then

$$
\sum_{l=1}^{n-1} P H_{l}(x)=\frac{P H_{n}(x)+P H_{n-1}(x)-P H_{0}(x)-P H_{1}(x)}{2 x} .
$$


Proof. For an integer $n \geq 2$ we have

$$
\begin{array}{rl}
\sum_{l=1}^{n-1} & P H_{l}(x)=P H_{1}(x)+P H_{2}(x)+\ldots+P H_{n-1}(x) \\
= & P_{1}(x)+\mathbf{i} P_{2}(x)+\varepsilon P_{3}(x)+\mathbf{h} P_{4}(x) \\
& +P_{2}(x)+\mathbf{i} P_{3}(x)+\varepsilon P_{4}(x)+\mathbf{h} P_{5}(x) \\
& +\cdots \\
& +P_{n-1}(x)+\mathbf{i} P_{n}(x)+\varepsilon P_{n+1}(x)+\mathbf{h} P_{n+2}(x) \\
= & P_{1}(x)+P_{2}(x)+\cdots+P_{n-1}(x) \\
& +\mathbf{i}\left(P_{2}(x)+P_{3}(x)+\cdots+P_{n}(x)+P_{1}(x)-P_{1}(x)\right) \\
& +\varepsilon\left(P_{3}(x)+P_{4}(x)+\cdots+P_{n+1}(x)+P_{1}(x)+P_{2}(x)\right. \\
& \left.-P_{1}(x)-P_{2}(x)\right) \\
& +\mathbf{h}\left(P_{4}(x)+P_{5}(x)+\cdots+P_{n+2}(x)+P_{1}(x)+P_{2}(x)+P_{3}(x)\right. \\
& \left.-P_{1}(x)-P_{2}(x)-P_{3}(x)\right) .
\end{array}
$$

Using (2.7) we obtain

$$
\begin{aligned}
& \sum_{l=1}^{n-1} P H_{l}(x)=\frac{P_{n}(x)+P_{n-1}(x)-1}{2 x} \\
& \quad+\mathbf{i}\left(\frac{P_{n+1}(x)+P_{n}(x)-1}{2 x}-P_{1}(x)\right) \\
& \quad+\varepsilon\left(\frac{P_{n+2}(x)+P_{n+1}(x)-1}{2 x}-P_{1}(x)-P_{2}(x)\right) \\
& \quad+\mathbf{h}\left(\frac{P_{n+3}(x)+P_{n+2}(x)-1}{2 x}-P_{1}(x)-P_{2}(x)-P_{3}(x)\right) .
\end{aligned}
$$

Bringing to the common denominator we have

$$
\begin{aligned}
& \sum_{l=1}^{n-1} P H_{l}(x)=\frac{P_{n}(x)+P_{n-1}(x)-1}{2 x} \\
& \quad+\mathbf{i}\left(\frac{P_{n+1}(x)+P_{n}(x)-1-2 x}{2 x}\right) \\
& \quad+\varepsilon\left(\frac{P_{n+2}(x)+P_{n+1}(x)-1-2 x-4 x^{2}}{2 x}\right) \\
& \quad+\mathbf{h}\left(\frac{P_{n+3}(x)+P_{n+2}(x)-1-2 x-4 x^{2}-2 x\left(4 x^{2}+1\right)}{2 x}\right)
\end{aligned}
$$


and finally

$$
\begin{aligned}
& \sum_{l=1}^{n-1} P H_{l}(x)=\frac{P_{n}(x)+\mathbf{i} P_{n+1}(x)+\varepsilon P_{n+2}(x)+\mathbf{h} P_{n+3}(x)}{2 x} \\
& \quad+\frac{P_{n-1}(x)+\mathbf{i} P_{n}(x)+\varepsilon P_{n+1}(x)+\mathbf{h} P_{n+2}(x)}{2 x} \\
& \quad+\frac{-(0+1)-\mathbf{i}(1+2 x)-\varepsilon\left(2 x+\left(4 x^{2}+1\right)\right)-\mathbf{h}\left(\left(4 x^{2}+1\right)+\left(8 x^{3}+4 x\right)\right)}{2 x} \\
& =\frac{P H_{n}(x)+P H_{n-1}(x)-P H_{0}(x)-P H_{1}(x)}{2 x} .
\end{aligned}
$$

Thus the Theorem is proved.

Theorem 16 ([3]). Let $n \geq 2$ be an integer. Then

$$
\sum_{l=1}^{n-1} Q_{l}(x)=\frac{Q_{n}(x)+Q_{n-1}(x)-2-2 x}{2 x} .
$$

Theorem 17. Let $n \geq 2$ be an integer. Then

$$
\sum_{l=1}^{n-1} Q H_{l}(x)=\frac{Q H_{n}(x)+Q H_{n-1}(x)-Q H_{0}(x)-Q H_{1}(x)}{2 x} .
$$

Proof. Using (2.8) and proceeding in the same way as in the Theorem 15 the result follows.

Next we shall give the generating function for Pell hybrinomials.

Theorem 18. The generating function for Pell hybrinomial sequence $\left\{P H_{n}(x)\right\}$ is

$$
G(t)=\frac{\mathbf{i}+\varepsilon \cdot(2 x)+\mathbf{h} \cdot\left(4 x^{2}+1\right)+(1+\varepsilon+\mathbf{h} \cdot(2 x)) t}{1-2 x t-t^{2}} .
$$

Proof. Assume that the generating function of the Pell hybrinomial sequence $\left\{P H_{n}(x)\right\}$ has the form $G(t)=\sum_{n=0}^{\infty} P H_{n}(x) t^{n}$. Then

$$
G(t)=P H_{0}(x)+P H_{1}(x) t+P H_{2}(x) t^{2}+\ldots
$$

Multiply the above equality on both sides by $-2 x t$ and then by $-t^{2}$ we obtain

$$
-G(t) \cdot(2 x) t=-P H_{0}(x) \cdot(2 x) t-P H_{1}(x) \cdot(2 x) t^{2}-P H_{2}(x) \cdot(2 x) t^{3}-\ldots
$$




$$
-G(t) t^{2}=-P H_{0}(x) t^{2}-P H_{1}(x) t^{3}-P H_{2}(x) t^{4}-\ldots
$$

By adding these three equalities above, we will get

$$
G(t)\left(1-2 x t-t^{2}\right)=P H_{0}(x)+\left(P H_{1}(x)-P H_{0}(x) \cdot(2 x)\right) t
$$

since $P H_{n}(x)=2 x \cdot P H_{n-1}(x)+P H_{n-2}(x)$ (see (2.1)) and the coefficient of $t^{n}$, for $n \geq 2$, are equal to zero. Moreover, $P H_{0}(x)=\mathbf{i}+\varepsilon \cdot(2 x)+\mathbf{h} \cdot\left(4 x^{2}+1\right), P H_{1}(x)-$ $P H_{0}(x) \cdot(2 x)=1+\varepsilon+\mathbf{h} \cdot(2 x)$.

In the same way we obtain the generating function $g(t)$ for Pell-Lucas hybrinomials.

Theorem 19. The generating function for the Pell-Lucas hybrinomial sequence $\left\{Q H_{n}(x)\right\}$ is

$$
g(t)=\frac{Q H_{0}(x)+\left(Q H_{1}(x)-Q H_{0}(x) \cdot(2 x)\right) t}{1-2 x t-t^{2}},
$$

where $Q H_{0}(x)=2+\mathbf{i} \cdot(2 x)+\varepsilon \cdot\left(4 x^{2}+2\right)+\mathbf{h} \cdot\left(8 x^{3}+6 x\right)$,

and $Q H_{1}(x)-Q H_{0}(x) \cdot(2 x)=-2 x+2 \mathbf{i}+\varepsilon \cdot(2 x)+\mathbf{h} \cdot\left(4 x^{2}+2\right)$.

We will give the matrix representation of Pell hybrinomials.

Theorem 20. Let $n \geq 0$ be an integer. Then

$$
\left[\begin{array}{ll}
P H_{n+2}(x) & P H_{n+1}(x) \\
P H_{n+1}(x) & P H_{n}(x)
\end{array}\right]=\left[\begin{array}{ll}
P H_{2}(x) & P H_{1}(x) \\
P H_{1}(x) & P H_{0}(x)
\end{array}\right] \cdot\left[\begin{array}{ll}
2 x & 1 \\
1 & 0
\end{array}\right]^{n} .
$$

Proof. (by induction on $n$ )

If $n=0$ then assuming that the matrix to the power 0 is the identity matrix the result is obvious. Now suppose that for any $n \geq 0$ holds

$$
\left[\begin{array}{ll}
P H_{n+2}(x) & P H_{n+1}(x) \\
P H_{n+1}(x) & P H_{n}(x)
\end{array}\right]=\left[\begin{array}{ll}
P H_{2}(x) & P H_{1}(x) \\
P H_{1}(x) & P H_{0}(x)
\end{array}\right] \cdot\left[\begin{array}{ll}
2 x & 1 \\
1 & 0
\end{array}\right]^{n} .
$$

We shall show that

$$
\left[\begin{array}{ll}
P H_{n+3}(x) & P H_{n+2}(x) \\
P H_{n+2}(x) & P H_{n+1}(x)
\end{array}\right]=\left[\begin{array}{ll}
P H_{2}(x) & P H_{1}(x) \\
P H_{1}(x) & P H_{0}(x)
\end{array}\right] \cdot\left[\begin{array}{ll}
2 x & 1 \\
1 & 0
\end{array}\right]^{n+1} .
$$

By simple calculation using induction's hypothesis we have

$$
\begin{gathered}
{\left[\begin{array}{ll}
P H_{2}(x) & P H_{1}(x) \\
P H_{1}(x) & P H_{0}(x)
\end{array}\right] \cdot\left[\begin{array}{ll}
2 x & 1 \\
1 & 0
\end{array}\right]^{n} \cdot\left[\begin{array}{cc}
2 x & 1 \\
1 & 0
\end{array}\right]} \\
=\left[\begin{array}{ll}
P H_{n+2}(x) & P H_{n+1}(x) \\
P H_{n+1}(x) & P H_{n}(x)
\end{array}\right] \cdot\left[\begin{array}{ll}
2 x & 1 \\
1 & 0
\end{array}\right] \\
=\left[\begin{array}{ll}
2 x \cdot P H_{n+2}(x)+P H_{n+1}(x) & P H_{n+2}(x) \\
2 x \cdot P H_{n+1}(x)+P H_{n}(x) & P H_{n+1}(x)
\end{array}\right] \\
=\left[\begin{array}{ll}
P H_{n+3}(x) & P H_{n+2}(x) \\
P H_{n+2}(x) & P H_{n+1}(x)
\end{array}\right],
\end{gathered}
$$


which ends the proof.

In the same way we obtain the matrix representation for Pell-Lucas hybrinomials.

Theorem 21. Let $n \geq 0$ be an integer. Then

$$
\left[\begin{array}{ll}
Q H_{n+2}(x) & Q H_{n+1}(x) \\
Q H_{n+1}(x) & Q H_{n}(x)
\end{array}\right]=\left[\begin{array}{ll}
Q H_{2}(x) & Q H_{1}(x) \\
Q H_{1}(x) & Q H_{0}(x)
\end{array}\right] \cdot\left[\begin{array}{ll}
2 x & 1 \\
1 & 0
\end{array}\right]^{n} .
$$

COMPLIANCE WITH ETHICAL STANDARDS

Conflict of Interest: The authors declare that they have no conflict of interest.

\section{REFERENCES}

[1] A. F. Horadam, "Pell identities." Fibonacci Quart., vol. 9, no. 3, pp. 245-263, 1971.

[2] A. F. Horadam, "Minmax Sequences for Pell Numbers." Applications of Fibonacci Numbers, vol. 6, pp. 231-249, 1996.

[3] A. F. Horadam and B. J. M. Mahon, "Pell and Pell-Lucas Polynomials." Fibonacci Quart., vol. 23, no. 1, pp. 7-20, 1985.

[4] T. Horzum and E. G. Kocer, "On Some Properties of Horadam Polynomials." Int. Math. Forum, vol. 25, no. 4, pp. 1243-1252, 2009.

[5] T. Koshy, Pell and Pell-Lucas Numbers with Applications. New York: Springer, 2014.

[6] M. Özdemir, "Introduction to Hybrid Numbers." Adv. Appl. Clifford Algebr., vol. 28, 2018, doi: 10.1007/s00006-018-0833-3.

[7] A. Szynal-Liana, "The Horadam hybrid numbers." Discuss. Math. Gen. Algebra Appl., vol. 38, no. 1, pp. 91-98, 2018, doi: 10.7151/dmgaa.1287.

[8] A. Szynal-Liana and I. Włoch, "On Pell and Pell-Lucas Hybrid Numbers." Comment. Math. Prace Mat., vol. 58, no. 1-2, pp. 11-17, 2018, doi: 10.14708/cm.v58i1-2.6364.

Authors' addresses

\section{Mirosław Liana}

Rzeszow University of Technology, The Faculty of Management, al. Powstańców Warszawy 10, 35-959 Rzeszów, Poland

E-mail address: mlianaeprz.edu.pl

Anetta Szynal-Liana

Rzeszow University of Technology, The Faculty of Mathematics and Applied Physics, al. Powstańców Warszawy 12, 35-959 Rzeszów, Poland

E-mail address: aszynaleprz.edu.pl

\section{Iwona Włoch}

Rzeszow University of Technology, The Faculty of Mathematics and Applied Physics, al. Powstańców Warszawy 12, 35-959 Rzeszów, Poland

E-mail address: iwloch@prz.edu.pl 